\section{A) Check for updates}

Cite this: Nanoscale, 2022, 14, 3768

\title{
Multivalent non-covalent interactions lead to strongest polymer adhesion $\uparrow$
}

\author{
Max Lallemang, (D) a,b Leixiao Yu, (DD c Wanhao Cai, (D) ${ }^{c}$ Klaus Rischka, (D) d \\ Andreas Hartwig, (iD d,e Rainer Haag, (iD c Thorsten Hugel iD *a,b and \\ Bizan N. Balzer (iD *a,b,f
}

\begin{abstract}
Multivalent interactions play a leading role in biological processes such as the inhibition of inflammation or virus internalization. The multivalent interactions show enhanced strength and better selectivity compared to monovalent interactions, but they are much less understood due to their complexity. Here, we detect molecular interactions in the range of a few piconewtons to several nanonewtons and correlate them with the formation and subsequent breaking of one or several bonds and assign these bonds. This becomes possible by performing atomic force microcopy (AFM)-based single molecule force spectroscopy of a multifunctional polymer covalently attached to an AFM cantilever tip on a substrate bound polymer layer of the multifunctional polymer. Varying the $\mathrm{pH}$ value and the crosslinking state of the polymer layer, we find that bonds of intermediate strength (non-covalent), like coordination bonds, give the highest multivalent bond strength, even outperforming strong (covalent) bonds. At the same time, covalent bonds enhance the polymer layer density, increasing in particular the number of non-covalent bonds. In summary, we can show that the key for the design of stable and durable polymer coatings is to provide a variety of multivalent interactions and to keep the number of non-covalent interactions at a high level.
\end{abstract}

Received 19th December 2021, Accepted 9th February 2022

DOI: $10.1039 / \mathrm{d} 1 \mathrm{nr} 08338 \mathrm{~d}$

rsc.li/nanoscale acterizing individual constituents of multivalent bonds is still challenging because a wide range of forces and interaction lengths are involved. ${ }^{1}$

One important amino acid used in nature to form multivalent bonds is the catecholic 3,4-dihydroxy-L-phenylalanine (DOPA). It is for example used by blue mussels, which can bind in wet and salty conditions to inorganic as well as organic surfaces. ${ }^{2}$ Catechols can reversibly be oxidized to form quinones, which is highly electrophilic and can irreversibly crosslink with other catechol moieties., ${ }^{3,4}$ Crosslinking can occur via Michael addition of the free amine with the quinone group, via Schiff Base formation of the amine with the carbonyl group of the quinone or via quinone-quinone coupling. ${ }^{5,6}$ The oxidation of catechol to semi-quinone and finally to quinone is a crucial step for crosslinking of adhesives based on catechol rich compounds. This evidence can be used to synthesize and tailor mussel-inspired and catechol-based polymers with strong adhesion to surfaces in order to obtain universal/substrate-independent coatings with self-healing or antifouling properties under mild conditions. ${ }^{7-10}$

Here we used an amphiphilic, catechol-based and multifunctional block copolymer $\mathrm{Br} / \mathrm{N}_{3}-\mathrm{PG}-b / r$-P(Cat-Ph-A) (Fig. 1a), which has been used before to create well-defined polymer monolayers. ${ }^{11,12}$ This multifunctional molecule comprises sites for secondary modification and combines the concepts known from blood protein adsorption with those known from 


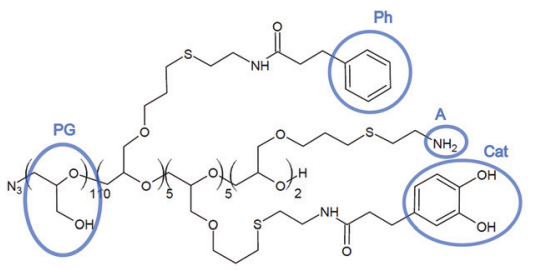

C

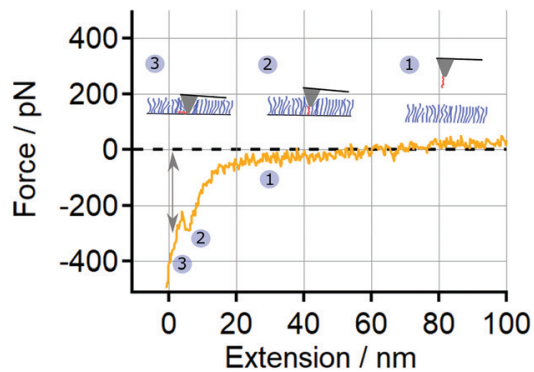

b

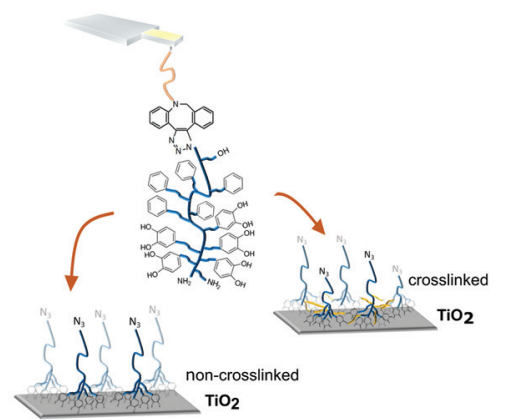

d

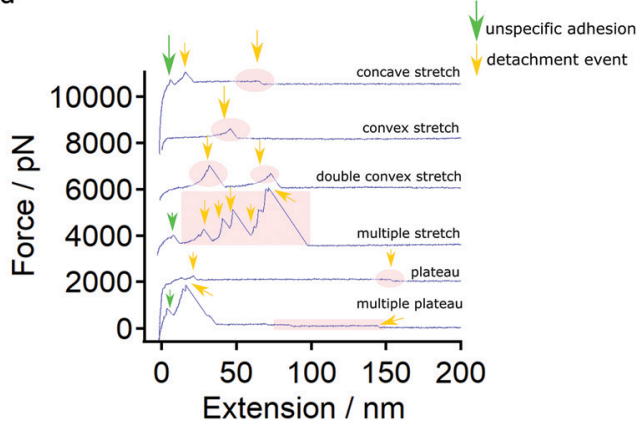

Fig. 1 Tools and experiments to quantify multivalent bond strength. (a) Scheme of a multivalent $N_{3}-P G_{110}-b-P\left(C a t_{5}-P h_{5}-A_{2}\right)$ molecule, showing a polyglycol (PG) block and catechol (Cat), phenyl (Ph) and amine (A) groups. (b) Scheme of a $P G_{110}-b-P\left(C_{1} t_{5}-P_{5}-A_{2}\right)$ molecular force sensor on noncrosslinked (left) and crosslinked (right) $N_{3}-P_{110}-b-P\left(C a t_{5}-P_{5}-A_{2}\right.$ ) layers on $T_{i} O_{2}$. (c) Approach curves for a $P G_{110}-b-P\left(C a t_{5}-P h_{5}-A_{2}\right) m_{0}$ mocular force sensor on a $\mathrm{N}_{3}-\mathrm{PG}_{110}-b-\mathrm{P}\left(\mathrm{Cat}_{5}-\mathrm{Ph}_{5}-\mathrm{A}_{2}\right)$ brush-like monolayer on $\mathrm{TiO}_{2}$ in $\mathrm{H}_{2} \mathrm{O}$ revealing a breakthrough event in the range of 100 to 400 pN (indicated by an arrow). (d) Characterization of the retract force-extension curves (offset by $2000 \mathrm{pN}$ ) on the $\mathrm{N}_{3}-\mathrm{PG}_{110}-b-\mathrm{P}\left(\mathrm{Cat}_{5}-\mathrm{Ph}_{5}-\mathrm{A}_{2}\right)$ layer, showing (from top to bottom) detachment events (marked by yellow arrows): concave stretch, convex stretch, double convex stretch, multiple stretches, plateau of constant force and multiple plateaus of constant force. The adhesion peak (marked by green arrows) at the beginning of the force-extension curves was not taken into account for evaluation, because it results from unspecific interaction of the whole AFM cantilever tip with the underlying sample.

mussel adhesion. An azide terminal group allows to perform a copper free type of the Huisgen reaction, which takes advantage of strain-promoted alkyne azide cycloaddition (SPAAC) using dibenzocyclooctine (DBCO) forming 1,2,3-triazoles. ${ }^{13-16}$ These reaction products are non-toxic, i.e., biocompatible, as well as efficient and practicable under mild conditions. ${ }^{17}$ Furthermore, the multifunctional molecule offers crosslinking via Schiff base formation or Michael addition.

However, the molecular understanding of the interplay between the different and multivalent types of interactions in such a coating, making it so unique, is still lacking because of the variety of different bonds. A better molecular understanding is crucial for a rational design of future polymer coatings.

Single molecule force spectroscopy (SMFS) with an Atomic Force Microscope (AFM) is a powerful method for studying the interactions between single molecules and surfaces or interfaces. ${ }^{18-21}$ The formation of isolated bonds like, e.g., S$\mathrm{Au}^{22} \mathrm{Si}-\mathrm{O}^{23}$ and $\mathrm{Si}-\mathrm{C}^{24}$ has been studied in detail by SMFS. Many experiments with a big diversity of catechol-based molecules demonstrated the strength of catechols and quinones on different substrates and in different $\mathrm{pH}$ and buffer conditions. There, detachment forces varied between approx. 50 and 1000 $\mathrm{pN}^{22,25-35}$ Additionally, SMFS on systems comprising multivalent interactions have been performed for different biological systems, obtaining detachment forces of up to 1000
$\mathrm{pN}^{36-42}$ However, the individual contributions to the overall force could not be resolved.

Here, we used covalent attachment of a $\mathrm{N}_{3}-\mathrm{PG}-b-\mathrm{P}$ (Cat-Ph-A) copolymer probe molecule to an AFM cantilever tip to study the multivalent interactions on the molecular level by SMFS. We used polymer layers consisting of the same $\mathrm{N}_{3}$-PG- $b$-P(CatPh-A) copolymer under different conditions and assign detachment forces to specific types of interaction. This allowed us to directly monitor the respective interactions, namely their multivalent bond strength and rupture force with piconewton resolution. Furthermore, we could show that the formation and rupture of strong (covalent) bonds cannot outperform the presence of interactions of low and intermediate strength (noncovalent). Altogether, this teaches us how to optimize the design of future multifunctional coatings using different types of molecular interactions and multivalency as a concept for creating robust coatings for various applications, especially in the field of chemistry, physics, material science and biomedicine.

\section{Materials and methods}

\section{RCA cleaning}

The glassware and tweezers were immersed in a RCA solution (Radio Corporation of America) having a volume ratio of 
5:1:1 of $\mathrm{H}_{2} \mathrm{O}$ (Purelab Chorus 1, Elga LabWater, Celle, Germany, 18.2 $\mathrm{M} \Omega \mathrm{cm}$ ), $\mathrm{NH}_{3}$ solution (Roth, Karlsruhe, Germany, 28.0-30.0\%), $\mathrm{H}_{2} \mathrm{O}_{2}$ (Sigma-Aldrich, St Louis, MO, USA, $\geq 30 \%$ ) at $70{ }^{\circ} \mathrm{C}$ for $40 \mathrm{~min}$. Afterwards, they were rinsed three times with $\mathrm{H}_{2} \mathrm{O}$, dried and stored at $120{ }^{\circ} \mathrm{C}$.

\section{Substrates}

Ti-wafers, named $\mathrm{TiO}_{2}$ in the following, were prepared by using 3 inches N/Phosphor doped Si wafers with a thickness of $380 \mu \mathrm{m}$, a 100 orientation and a resistance of $7.5 \Omega \mathrm{cm}(4 \mathrm{P} 219$ / 90, Silchem, Germany). A $50 \mathrm{~nm}$ thick Ti-layer was applied by a sputter process using a high-vacuum coater (Leica EM ACE600, Leica Microsystems GmbH, Wetzlar, Germany). The thickness was controlled during the sputter process (current $100 \mathrm{~mA}, \mathrm{Ar}$ pressure $0.01 \mathrm{mbar}$, working distance $50 \mathrm{~mm}$, tilt angle $20^{\circ}$ ) by a quartz crystal thickness measurement.

\section{Polymer}

$\mathrm{N}_{3}-\mathrm{PG}_{110}-b-\mathrm{P}\left(\mathrm{Cat}_{5}-\mathrm{Ph}_{5}-\mathrm{A}_{2}\right)$ with a molecular weight of $12 \mathrm{kDa}$ is an amphiphilic block copolymer used in this work (Fig. 1a). The polyglycol (PG) acts as a hydrophilic domain. Poly (allyl glycidyl ether) is functionalized by amine groups (A, 2 units), catechol groups (Cat, 5 units) and phenyl groups (Ph, 5 units). An azide group serves as a functional site for secondary modification. The contour length $(43 \mathrm{~nm})$ of the polymer was calculated by multiplying the number of polyethylene glycol (PEG) and PG groups $N$ by the length $l$ of one PEG or PG-repeat unit $(0.356 \mathrm{~nm}){ }^{43,44}$ Together with the triethoxysilane-PEG-DBCO (silane-PEG-DBCO, NANOCS, Boston, MA, USA) linker having a length of about $41 \mathrm{~nm}$, the final $\mathrm{PG}_{110}-b-\mathrm{P}\left(\mathrm{Cat}_{5}-\mathrm{Ph}_{5}-\mathrm{A}_{2}\right)$ molecular force sensor used in the SMFS experiments has a total contour length of about $84 \mathrm{~nm}$.

\section{Substrate and polymer layer preparation}

The $\mathrm{TiO}_{2}$-wafers were cleaned ultrasonically for $5 \mathrm{~min}$ first with $\mathrm{H}_{2} \mathrm{O}$ followed by $\mathrm{MeOH}$. Afterwards, the $\mathrm{TiO}_{2}$-wafers were cleaned with an oxygen plasma (15\% power, $0.1 \mathrm{mbar}, 20 \mathrm{~min}$, Diener Electronics, Germany). To prepare polymer layers, the cleaned slides were incubated for $2 \mathrm{~h}$ in a solution of $\mathrm{N}_{3}-\mathrm{PG}_{110^{-}}$ $b$ - $\mathrm{P}\left(\mathrm{Cat}_{5}-\mathrm{Ph}_{5}-\mathrm{A}_{2}\right)\left(1 \mathrm{mg} \mathrm{mL}{ }^{-1}\right)$ in a $\mathrm{pH} 6$ 3-( $N$-morpholino)propanesulfonic acid (MOPS, $0.1 \mathrm{M}$, Applichem $\mathrm{GmbH}$, Darmstadt, Germany) buffer at $22{ }^{\circ} \mathrm{C}^{11}$ The $\mathrm{pH}$ was adjusted by using a $\mathrm{pH}$ meter (LLG pH meter 7, Meckenheim, Germany) and solutions of $\mathrm{NaOH}(1.0 \mathrm{M})$ for the $\mathrm{pH} 8.6$ buffer and $\mathrm{HCl}$ $(1.0 \mathrm{M})$ for the $\mathrm{pH} 6.0$ buffer. Afterwards, the slides were rinsed with $\mathrm{H}_{2} \mathrm{O}$, followed by $\mathrm{MeOH}$ and finally dried by a $\mathrm{N}_{2}$ stream. To induce the crosslinking reaction, the slides were stored for $1 \mathrm{~h}$ in a solution of $\mathrm{K}_{2} \mathrm{~S}_{2} \mathrm{O}_{8}\left(10 \mathrm{mg} \mathrm{mL}^{-1}\right)$ in $\mathrm{pH} 8.6$ MOPS $(0.1 \mathrm{M})$ buffer at $22{ }^{\circ} \mathrm{C}^{11}$ After that, the slides were rinsed with $\mathrm{H}_{2} \mathrm{O}$, followed by $\mathrm{MeOH}$ and finally dried by a $\mathrm{N}_{2}$ stream. For control experiments with $\mathrm{SiO}_{2}, \mathrm{SiO}_{2}$-wafers were cleaned in ethanol for $5 \mathrm{~min}$ at $22{ }^{\circ} \mathrm{C}$ using a sonicator (Elmasonic S15, Elma, Singen, Germany).

\section{AFM cantilever tip functionalization}

The following processes have been optimized to ensure a proper passivation of the cantilever tip surface with $5 \mathrm{kDa}$ silane-PEG and the attachment of $\mathrm{PG}_{110}-b-\mathrm{P}\left(\mathrm{Cat}_{5}-\mathrm{Ph}_{5}-\mathrm{A}_{2}\right)$ to finally obtain detachment events in the SMFS experiment.

\section{$\mathrm{PG}_{110}-b-\mathrm{P}\left(\mathrm{Cat}_{5}-\mathrm{Ph}_{5}-\mathrm{A}_{2}\right)$ molecular force sensor}

For functionalization of cantilever tips with $\mathrm{N}_{3}-\mathrm{PG}_{110}-b-\mathrm{P}\left(\mathrm{Cat}_{5}-\right.$ $\mathrm{Ph}_{5}-\mathrm{A}_{2}$ ) which is schemed in Fig. $\mathrm{S} 1, \uparrow \mathrm{Si}_{3} \mathrm{~N}_{4}$ MLCT-BIO-DC cantilevers (Bruker AFM probes, Camarillo, CA, USA) were used. First, the cantilevers were activated with oxygen plasma (Diener Electronics, Germany) to gain hydroxyl groups on the surface of the cantilever tips. The MLCT-BIO-DC probes were treated for $2 \mathrm{~min}$ with $40 \%$ power at a pressure of $0.1 \mathrm{mbar}$. As a next step, a 5 kDa silane-PEG-DBCO (NANOCS, Boston, MA, USA) linker was bound to the cantilever tip. Due to the sensitivity of DBCO towards light, the following steps were performed in the dark. The cantilevers were incubated in a solution of silane-PEG-DBCO (PEG-DBCO) in toluene $1.25 \mathrm{mg}$ $\mathrm{mL}^{-1}, 2 \mathrm{~h}$ ) at $22^{\circ} \mathrm{C}$. Then, the PEGylated cantilevers were rinsed in toluene, EtOH, $\mathrm{H}_{2} \mathrm{O}$ and were finally incubated in a solution of $\mathrm{N}_{3}-\mathrm{PG}_{110}-b-\mathrm{P}\left(\mathrm{Cat}_{5}-\mathrm{Ph}_{5}-\mathrm{A}_{2}\right)$ in a $1: 1$ solution of PBS buffer (Dulbecco's Phosphate Buffered Saline, pH 7.2, SigmaAldrich, USA) and DMSO (1.25 $\left.\mathrm{mg} \mathrm{mL}^{-1}, 24 \mathrm{~h}\right)$ at $22{ }^{\circ} \mathrm{C}$ to induce the copper-free click chemistry of the azide group of $\mathrm{N}_{3}-\mathrm{PG}_{110}-b-\mathrm{P}\left(\mathrm{Cat}_{5}-\mathrm{Ph}_{5}-\mathrm{A}_{2}\right)$ and DBCO. After final rinsing, the cantilevers were stored in HEPES (Sigma-Aldrich, St Louis, MO, USA) buffer ( $\mathrm{pH} 7.0,50 \mathrm{mM} \mathrm{NaCl}$ ) at $4{ }^{\circ} \mathrm{C}$ until use in the AFM experiment. For each functionalization, control cantilevers (carrying the $5 \mathrm{kDa}$ silane-PEG-DBCO only) were additionally prepared by the same procedure incubating the cantilevers in the pure solvent (DMSO) instead of $\mathrm{N}_{3}-\mathrm{PG}_{110}-b-\mathrm{P}\left(\mathrm{Cat}_{5}-\mathrm{Ph}_{5}-\right.$ $A_{2}$ ).

\section{AFM-based force spectroscopy}

All SMFS experiments were performed with a Cypher ES (Asylum Research, an Oxford Instruments company, Santa Barbara, CA, USA). Before each SMFS experiment, the inverse optical lever sensitivity (InvOLS) was determined by fitting a linear function to the repulsive region of a force-extension curve. In order to reduce errors, the determination of the InvOLS was performed by using an average of at least five individual InvOLS values. The spring constant of the cantilever was determined by the thermal noise method. ${ }^{45}$

$\mathrm{PG}_{110}-b-\mathrm{P}\left(\mathrm{Cat}_{5}-\mathrm{Ph}_{5}-\mathrm{A}_{2}\right)$ on the $\mathrm{N}_{3}-\mathrm{PG}_{110}-b-\mathrm{P}\left(\mathrm{Cat}_{5}-\mathrm{Ph}_{5}-\mathrm{A}_{2}\right)$ layer on $\mathrm{TiO}_{2}$ took place in ultrapure $\mathrm{H}_{2} \mathrm{O}$, MOPS buffer $\mathrm{pH} 6.0$ $(0.1 \mathrm{M})$ and MOPS buffer $\mathrm{pH} 8.6$ (0.1 M). $\mathrm{PG}_{110}-b-\mathrm{P}\left(\mathrm{Cat}_{5}-\mathrm{Ph}_{5^{-}}\right.$ $\mathrm{A}_{2}$ ) and silane-PEG-DBCO were probed on $\mathrm{SiO}_{2}$ in ultrapure water.

SMFS parameters were defined as follows: force distance, $1 \mu \mathrm{m}$; velocity, $1 \mu \mathrm{m} \mathrm{s}^{-1}$; contact force, 50 to $3000 \mathrm{pN}$; sampling rate, $5 \mathrm{kHz}$; contact time towards the surface, $0-10 \mathrm{~s}$ and temperature, $25^{\circ} \mathrm{C}$. To reduce the influence of local sample variations, force-extension curves were recorded in a grid-like manner with $10 \cdot 10$ points covering $20 \cdot 20 \mu \mathrm{m}^{2}$ (force maps). At 
least two force maps were obtained per cantilever. Prior to a series of measurements, at least one control cantilever (same procedure but the incubation took place in pure solvent instead of the target molecule) was measured on different surface spots to verify a contamination-free functionalization. Once the control cantilevers (silane-PEG-DBCO on $\mathrm{SiO}_{2}$ ) showed an absence of stretching events or plateaus of constant force, the $\mathrm{PG}_{110}-b-\mathrm{P}\left(\mathrm{Cat}_{5}-\mathrm{Ph}_{5}-\mathrm{A}_{2}\right)$ functionalized cantilevers were measured, respectively. For data evaluation, a self-programmed evaluation software based on Igor Pro (Wavemetrics, Portland, OR, USA) was used. For the analysis of detachment forces, all detachment events such as stretches and plateaus were taken into account, except the adhesion peak at the beginning of the force-extension curves (Fig. 1d), resulting from unspecific interactions of the whole AFM cantilever tip and the surface.

OriginPro 2019 (OriginLab, Northampton, MA, USA) was used for data presentation and to test the significance. The latter was performed by an unpaired two-tailed Mann-Whitney $U$ test was performed with for the force values. The null hypothesis in the Mann-Whitney $U$ test is that $X$ and $Y$ are two random selected values from two populations, the probability of $Y$ being greater than $X$ is equal to the probability of $X$ being greater than $Y$. The level of significance was set to 0.05 .

\section{AFM-based scratching}

The thickness of the $\mathrm{N}_{3}-\mathrm{PG}_{110}-b-\mathrm{P}\left(\mathrm{Cat}_{5}-\mathrm{Ph}_{5}-\mathrm{A}_{2}\right)$ polymer layer was determined by AFM scratching experiments on a Cypher ES (Asylum Research, an Oxford Instruments company, Santa Barbara, CA, USA) using AC240TS cantilevers (Olympus, Japan). AFM imaging of those polymer layers was performed in $\mathrm{pH}$ 6.0 MOPS buffer $(0.1 \mathrm{M})$ at a temperature of $25^{\circ} \mathrm{C}$. The first image of each sample was taken in the intermittentcontact mode with a scan size of $15 \cdot 15 \mu \mathrm{m}^{2}$. Then, the surface was scratched in contact mode with a scan size of $1.1 \mu \mathrm{m}^{2}$ and an applied force (trigger force) of $648 \mathrm{nN}$. The imaging mode was switched back again to intermittent-contact mode for further imaging. All images were taken at a scan rate of $2 \mathrm{~Hz}$. The depth of the hole, created by the cantilever tip in contact mode, was used to determine the presence and thickness of the polymer layer on the respective substrate.

\section{Results}

\section{Characterization of multivalent interactions by SMFS}

We used a $\mathrm{PG}_{110}-b-\mathrm{P}\left(\mathrm{Cat}_{5}-\mathrm{Ph}_{5}-\mathrm{A}_{2}\right)$ molecule (Fig. 1a) covalently bound to the AFM cantilever tip (Fig. $1 \mathrm{~b}$ and $\mathrm{S} 1 \dagger$ ) as a molecular force sensor. The molecular force sensor was measured on $\mathrm{N}_{3}-\mathrm{PG}_{110}-b-\mathrm{P}\left(\mathrm{Cat}_{5}-\mathrm{Ph}_{5}-\mathrm{A}_{2}\right)$ layers on $\mathrm{TiO}_{2}$ (Fig. $1 \mathrm{~b}$ ) to understand bond formation and rupture. The presence and thickness of this layers on $\mathrm{TiO}_{2}$ have been tested by contact angle and AFM imaging experiments (Fig. S2 and S3†).

The covalent attachment of one single polymer to a cantilever is crucial for single molecule force experiments. It enables a high reproducibility when performing force spectroscopy with a certain polymer on a specific cantilever tip. In our case, the successful attachment of one single polymer has been verified by measuring the $\mathrm{PG}_{110}-b-\mathrm{P}\left(\mathrm{Cat}_{5}-\mathrm{Ph}_{5}-\mathrm{A}_{2}\right)$ molecular force sensor on $\mathrm{SiO}_{2}$ showing single molecule detachment events (Fig. S4 $\dagger$ ). Control measurements with silane-PEG-DBCO (5 kDa) show that these detachment events are indeed caused by the $\mathrm{PG}_{110^{-}}-\mathrm{P}-\mathrm{P}\left(\mathrm{Cat}_{5}-\mathrm{Ph}_{5}-\mathrm{A}_{2}\right)$ and not by unspecific adhesion between AFM cantilever tip and the $\mathrm{SiO}_{2}$ surface (Fig. S5 $\dagger$ ).

Finally, a variation of the contact force and contact time (Fig. S6 and S7 $\dagger$ ) revealed that a contact force of $500 \mathrm{pN}$ and a contact time of $1 \mathrm{~s}$ was ideal to obtain reproducible indentation into the layer (breakthrough) and detachment events as depicted in Fig. 1c and d.

The variety of the possible interactions between the $\mathrm{PG}_{110^{-}}$ $b$ - $\mathrm{P}\left(\mathrm{Cat}_{5}-\mathrm{Ph}_{5}-\mathrm{A}_{2}\right)$ molecular force sensor and the $\mathrm{N}_{3}-\mathrm{PG}_{110}-b-\mathrm{P}$ $\left(\mathrm{Cat}_{5}-\mathrm{Ph}_{5}-\mathrm{A}_{2}\right)$ layer is reflected by the obtained force-extension traces. We classified them into concave stretch, convex stretch, double (multiple) convex stretches, single plateau of constant force and multiple plateaus of constant force (Fig. 1d).

In order to assign the detachment events to specific interactions, we performed SMFS measurements under different conditions. First in MOPS buffer at pH 6.0 (0.1 M). Then, the buffer was exchanged to MOPS buffer $\mathrm{pH} 8.6(0.1 \mathrm{M})$ for the next SMFS experiments. Second, a crosslinked $\mathrm{N}_{3}-\mathrm{PG}_{110}-b-\mathrm{P}$ $\left(\mathrm{Cat}_{5}-\mathrm{Ph}_{5}-\mathrm{A}_{2}\right)$ layer was prepared using the oxidant $\left(\mathrm{K}_{2} \mathrm{~S}_{2} \mathrm{O}_{8}\right)$ in MOPS buffer pH $8.6(0.1 \mathrm{M})$ according to the protocol of $\mathrm{Yu}$ et al. ${ }^{11}$ Then, the crosslinked polymer layer was taken for SMFS experiments in MOPS buffer pH 8.6 (0.1 M).

Fig. 2a illustrates a representative force-extension curve (condition: $\mathrm{pH}$ 8.6) that comprises several detachment events. Further force-extension curves of that type are shown in Fig. $\mathrm{S} 8 \dagger$ (condition: $\mathrm{pH} 8.6$ crosslinked). Fig. $2 \mathrm{~b}$ represents the different measured detachment forces, resulting mostly from convex stretches (see Table $\mathrm{S} 1 \dagger$ for a quantification). The median and average forces increased from $\mathrm{pH} 6.0$ to $\mathrm{pH} 8.6$ crosslinked. An unpaired two-tailed Mann-Whitney $U$ test (with a level of significance of 0.05 , see Materials and Methods) showed that the difference between $\mathrm{pH} 6.0$ and $\mathrm{pH}$ 8.6 and between pH 8.6 crosslinked and the other two conditions ( $\mathrm{pH} 6.0$ and $\mathrm{pH}$ 8.6) was significant, respectively. In general, the forces of $\mathrm{PG}_{110}-b-\mathrm{P}\left(\mathrm{Cat}_{5}-\mathrm{Ph}_{5}-\mathrm{A}_{2}\right)$ on $\mathrm{N}_{3}-\mathrm{PG}_{110}-b-\mathrm{P}$ $\left(\mathrm{Cat}_{5}-\mathrm{Ph}_{5}-\mathrm{A}_{2}\right)$ exceed the forces on $\mathrm{SiO}_{2}$ (Fig. $\mathrm{S} 4 \dagger$ ) by a factor of more than 10, demonstrating the strength of multivalent interactions.

\section{Assignment of different interactions of the multivalent system}

Fig. 2c shows the possible types of interactions between the $\mathrm{PG}_{110}-b-\mathrm{P}\left(\mathrm{Cat}_{5}-\mathrm{Ph}_{5}-\mathrm{A}_{2}\right)$ molecular force sensor and the $\mathrm{N}_{3}$ $\mathrm{PG}_{110}-b-\mathrm{P}\left(\mathrm{Cat}_{5}-\mathrm{Ph}_{5}-\mathrm{A}_{2}\right)$ layer. These can be van der Waals (vdW) interaction (depicted in purple), hydrogen bonds ${ }^{44}$ (depicted in green) between hydrogen atoms of the catechol (5 groups) and oxygen of $\mathrm{TiO}_{2},{ }^{46,47} \pi-\pi$ stacking (depicted in red) between the aromatic rings of the catechols (5 groups), phenyls (5 groups) and DBCO (Fig. 2c, left). ${ }^{21,48}$ Coordination bonds (depicted in blue) can be formed between oxygen atoms of quinone groups (5 groups) and Ti atoms (Fig. 2c, middle). 

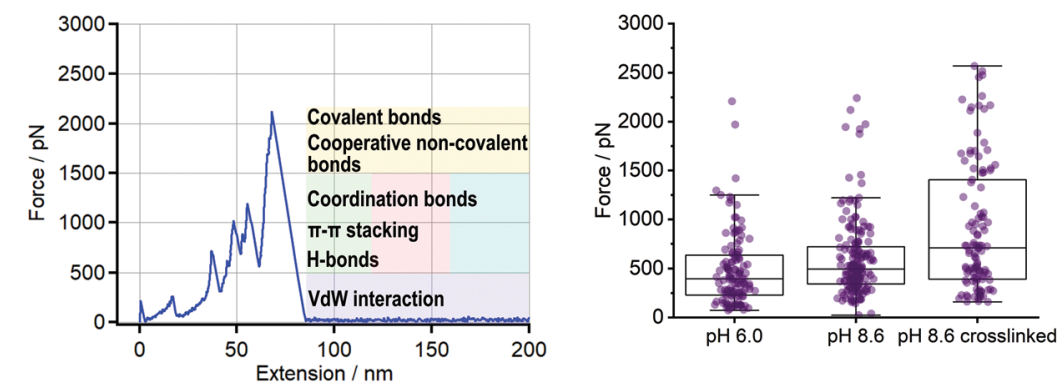

C
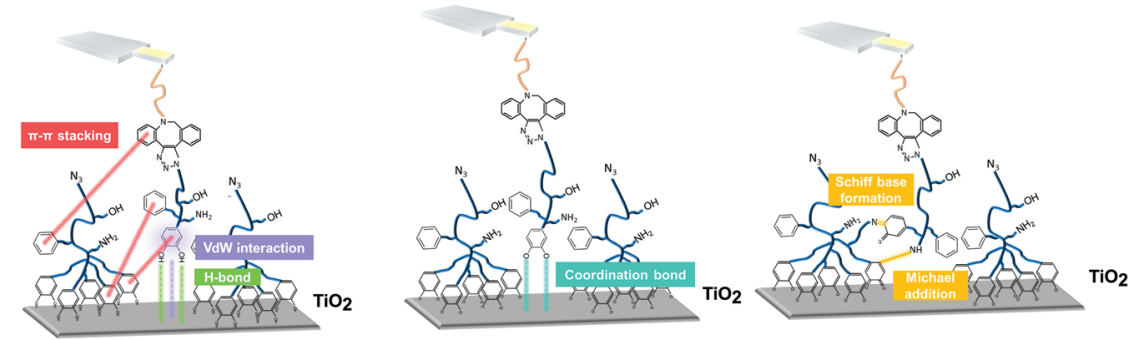

Fig. 2 Dependence of multivalent interactions on $\mathrm{pH}$ value and crosslinking. (a) An exemplary force-extension curve for a $P G_{110}-b-P\left(C a t_{5}-P h_{5}-A_{2}\right)$ molecular force sensor on a non-crosslinked $\mathrm{N}_{3}-\mathrm{PG}_{110}-b-\mathrm{P}\left(\mathrm{Cat}_{5}-\mathrm{Ph}_{5}-\mathrm{A}_{2}\right.$ ) layer on $\mathrm{TiO}_{2}$ (condition: $\mathrm{pH}$ 8.6). The first peak at zero extension was due to unspecific adhesion of the whole AFM cantilever tip and the polymer layer and has been omitted for evaluation. (b) The force distributions given by box plots are shown for $\mathrm{pH} 6.0, \mathrm{pH} 8.6$ and pH 8.6 crosslinked, revealing an increase of the median force with increasing $\mathrm{pH}$ and crosslinking of the polymer layer. The whiskers correspond to the lower quartile Q1 - 1.5.interquartile range (minimum) and the upper quartile Q3 + 1.5.interquartile range (maximum) or the last value within that range, respectively. 119 detachment events were obtained for $\mathrm{pH} 6.0,171$ for $\mathrm{pH} 8.6$ and 110 for $\mathrm{pH} 8.6$ crosslinked. For pH 6.0, the median value corresponds to $396 \mathrm{pN}$, the lower quartile Q1 and the upper quartile Q3 have values of $228 \mathrm{pN}$ and $638 \mathrm{pN}$, respectively. For $\mathrm{pH} 8.6$, the median value corresponds to $495 \mathrm{pN}$, the lower quartile Q1 and the upper quartile Q3 have values of $343 \mathrm{pN}$ and $719 \mathrm{pN}$, respectively. For $\mathrm{pH} 8.6$ crosslinked, the median has a value of $707 \mathrm{pN}$, the lower quartile Q1 and the upper quartile Q3 correspond to $391 \mathrm{pN}$ and $1406 \mathrm{pN}$, respectively. The average detachment force corresponds to $(491 \pm 373) \mathrm{pN}$ for pH $6.0,(598 \pm 389) \mathrm{pN}$ for pH 8.6 and $(903 \pm$ 655) $\mathrm{pN}$ for $\mathrm{pH} 8.6$ crosslinked. The errors correspond to the standard deviation. (c) Scheme of the different types of possible interactions.

Finally, strong interaction between amines (2 groups) and quinone groups (5 groups), accounting for the formation of a covalent $\mathrm{N}=\mathrm{C}$ (Schiff base reaction) or $\mathrm{NH}-\mathrm{C}$ (Michael addition) bond with a subsequent rupture event (Fig. 2c, right) are also possible.

In principle, the detachment events in the force-extension curves could directly be assigned to the respective interactions, but the multitude of possible interactions makes this difficult. Therefore, we also evaluated the frequency of the detachment forces and their positions. Fig. 3a shows a summary of the results, which guided us in assigning the different interactions. We subdivided the distribution of the forces into three different regions (R1, R2 and R3). Forces in region 1 (R1, up to $500 \mathrm{pN}$ ) probably originate from low force interactions like vdW interaction (in purple, Fig. $2 \mathrm{c}$ ). But also $\pi-\pi$ stacking (in red, Fig. 2c), hydrogen bonds (in green, Fig. 2c) and coordination bonds (depicted in blue) will already contribute here. This is based on literature values for the single bond types: forces of vdW interactions, range between 21 to $100 \mathrm{pN}^{48-50}$ The forces of hydrogen bonds range from a few to several tens of pN depending on the exact geometry of the molecules and direction of force application. ${ }^{51,52}$ Altogether, 232 hydrogen bonds (PEG backbone and $\mathrm{OH}$ side groups) could be formed at maximum, which then would exceed $500 \mathrm{pN}$ by far (see regions 2 and 3). Additionally, phenyl and catechol groups could contribute via $\pi-\pi$ stacking where the forces are reported to be between 2 and $8 \mathrm{pN}$ for DNA base pair unstacking and approx. 70 to $80 \mathrm{pN}$ for strong donor-acceptor $\pi$-interactions in $\mathrm{H}_{2} \mathrm{O}$, respectively. ${ }^{53,54}$ In our multivalent system, there are 12 possibilities for $\pi-\pi$ stacking (5·phenyl, $5 \cdot$ catechol, $2 \cdot$ phenyl from DBCO) leading to forces in the range of several $100 \mathrm{pN}$. In case of cooperative effects (non-linear increase of force with number of bonds) a further increase of the observed force is possible. ${ }^{55-57}$ Finally, the interaction between a single catechol and $\mathrm{TiO}_{2}$ is 300 to $1000 \mathrm{pN}$, according to the literature. ${ }^{25,26,29}$ For more basic $\mathrm{pH}$ values (e.g., $\mathrm{pH}=8.6)$, more and more quinone groups are formed, ${ }^{58,59}$ which interact with $\mathrm{TiO}_{2}$ via coordination bonds with forces of 50 to $300 \mathrm{pN}^{25,26,29}$ Therefore, also a catechol- $\mathrm{TiO}_{2}$ bond can be found in region 1 , although rarely.

In force region 2 (R2, 500 to $1500 \mathrm{pN}$ ), previous studies showed that multiple bonds between catechol and $\mathrm{TiO}_{2}$ are likely to be formed and show forces of above $500 \mathrm{pN}$, depending on the polymer architecture and the time in contact between the polymer and the underlying surface. ${ }^{25,33,35}$ Therefore, catechol, quinone, amine and phenyl groups might mostly contribute to the forces in that region. In other words, several catechol groups of the $\mathrm{PG}_{110}-b-\mathrm{P}\left(\mathrm{Cat}_{5}-\mathrm{Ph}_{5}-\mathrm{A}_{2}\right)$ molecular 
a
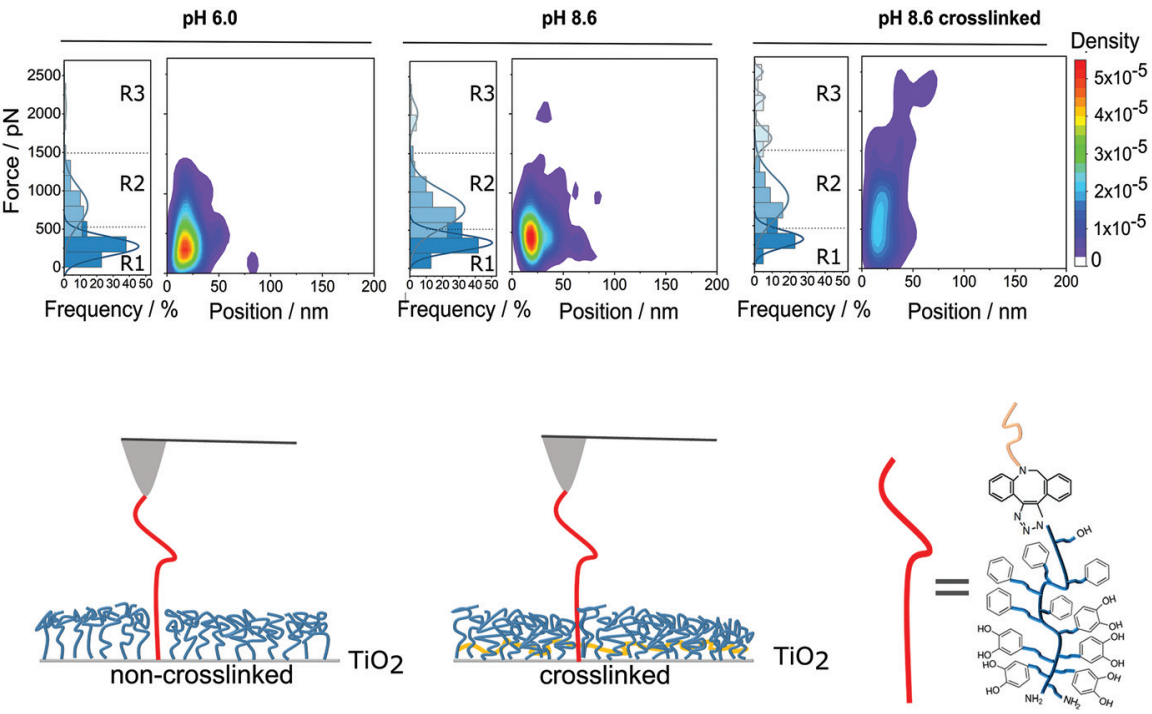

Fig. 3 Classification of multivalent interactions. (a) Heat maps of detachment events for the $P G_{110}-b-P\left(C a t_{5}-P h_{5}-A_{2}\right)$ molecular force sensor on the non-crosslinked and crosslinked $\mathrm{N}_{3}-\mathrm{PG}_{110}-b-\mathrm{P}\left(\mathrm{Cat}_{5}-\mathrm{Ph}_{5}-\mathrm{A}_{2}\right)$ layers on $\mathrm{TiO}_{2}$ under the different preparation conditions of the polymer layer, as given in Fig. $2 \mathrm{~b}$. The frequency of the detachment events as well as the heat maps of the detachment force vs. the detachment position are shown. There the density corresponds to the probability of the detected force and position values. The detachment forces are subdivided into different regions R1, R2 and R3 (see main text for details). (b) Scheme of non-crosslinked and crosslinked (in yellow) polymer layers for incorporation of the molecular force sensor into the polymer film.

force sensor could interact simultaneously with the $\mathrm{TiO}_{2}$ surface and the $\mathrm{N}_{3}-\mathrm{PG}_{110}-b-\mathrm{P}\left(\mathrm{Cat}_{5}-\mathrm{Ph}_{5}-\mathrm{A}_{2}\right)$ layer on $\mathrm{TiO}_{2}$. Once several of the five catechol/quinone groups of the molecular force sensor form coordination bonds with $\mathrm{TiO}_{2}$, they could even deliver forces above $1000 \mathrm{pN}$. Thus, force region 2 most likely originates from multivalent interaction of catechols/quinones and $\mathrm{TiO}_{2}$ in addition to interactions from R1. In particular, a multitude of hydrogen bonds resulting from the PEG backbone and $\mathrm{OH}$ side groups likely contribute significantly to the forces observed in the region $\mathrm{R} 2$.

Finally, force region 3 (R3), corresponding to the third populations in the histograms, shows forces higher than 1500 $\mathrm{pN}$. This force region (R3) is only significantly populated in the case of the crosslinked polymer layer. There, the detachment events could either result from the breakage of several non-covalent bonds, such as given above, in a possibly cooperative fashion, or due to covalent bonds.

Fig. $3 \mathrm{~b}$ shows the schematic comparison between the noncrosslinked (Fig. 3b, left) and crosslinked (Fig. 3b, right) polymer layer. ${ }^{11}$ In particular, crosslinking of the $\mathrm{N}_{3}-\mathrm{PG}_{110}-b-\mathrm{P}$ $\left(\mathrm{Cat}_{5}-\mathrm{Ph}_{5}-\mathrm{A}_{2}\right)$ layer could enhance the density of chains ${ }^{11}$ (which is consistent with the measured decrease in layer thickness, see Fig. S3 $\dagger$ ). Thus, the $\mathrm{PG}_{110}-b-\mathrm{P}\left(\mathrm{Cat}_{5}-\mathrm{Ph}_{5}-\mathrm{A}_{2}\right)$ molecular force sensor can interact with more neighboring chains of the polymer layer possibly leading to more multivalent interactions and more covalent bonds. The higher density of the crosslinked polymer layer might support the formation of up to five catechol/quinone coordination bonds in a cooperative way and also lead to forces higher than $1500 \mathrm{pN}$.

Furthermore, with increasing $\mathrm{pH}$, quinone groups are increasingly formed both, in the molecular force sensor and the polymer layer on $\mathrm{TiO}_{2}$. Quinone groups have a higher reactivity and are prone to attacks by nucleophiles and therefore, the formation of a covalent $\mathrm{N}=\mathrm{C}$ (Schiff base reaction) or NH-C (Michael addition) bond with a subsequent rupture is likely. ${ }^{60}$ Finally, by contacting the $\mathrm{PG}_{110}-\mathrm{b}-\mathrm{P}\left(\mathrm{Cat}_{5^{-}}\right.$ $\left.\mathrm{Ph}_{5}-\mathrm{A}_{2}\right)$ molecular force sensor and the $\mathrm{N}_{3}-\mathrm{PG}_{110}-b-\mathrm{P}\left(\mathrm{Cat}_{5}-\mathrm{Ph}_{5^{-}}\right.$ $\mathrm{A}_{2}$ ) layer, a high pressure of approx. 0.4 MPa is applied (taking an indentation force of $500 \mathrm{pN}$ and a nominal tip radius of $20 \mathrm{~nm}$ ). This is sufficient to form pressure induced covalent bonds. ${ }^{61}$

Also, the highest forces (R3) had the highest position values (Fig. 3a, right). Thus, the highest interactions resulted from the very end of the polymer, namely the quinone and amine groups which are possibly involved in the formation of covalent bonds. In theory, the maximum number of covalent bonds per $\mathrm{PG}_{110}-b-\mathrm{P}\left(\mathrm{Cat}_{5}-\mathrm{Ph}_{5}-\mathrm{A}_{2}\right)$ polymer amounts to 7 $\left(2 \cdot \mathrm{NH}_{2}, 5 \cdot\right.$ catechol, which could also form coordination bonds). This would lead to a maximum force of more than 7 $\mathrm{nN}$, when just assuming additivity of the respective interaction without cooperative effects. This has not been observed here and underlines that the simultaneous formation of multiple covalent bonds between molecular force sensor and polymer layer within seconds is unlikely.

Additionally, the increase of possible bonding positions of the molecular force sensor to the polymer layer may cause a misalignment between pulling direction and bond rupture direction. As discussed by Schoeler et al., ${ }^{62}$ this misalignment could also lead to a higher bond rupture force.

The difference between $\mathrm{pH} 8.6$ and $\mathrm{pH} 8.6$ crosslinked is the crosslinking of the polymer layer, resulting in a higher density. Therefore, most likely, more bonds of any kind are 
formed, resulting in the observed increase of molecular rupture events in R3, likely in a cooperative manner.

\section{Discussion}

\section{Multivalent bond strength and high density as key factor for stable and durable coatings}

After identifying and quantifying all the different interactions that we could possibly observe, we can draw a conclusion on the question which type of interaction is mostly responsible for the robustness of $\mathrm{N}_{3}-\mathrm{PG}_{110}-b-\mathrm{P}\left(\mathrm{Cat}_{5}-\mathrm{Ph}_{5}-\mathrm{A}_{2}\right)$ layer on $\mathrm{TiO}_{2}$. To that aim, we determine a new quantity, namely the multivalent bond strength, which is the product of the force and its relative frequency of occurrence. Fig. 4 shows the highest level of multivalent bond strength for region R2 for every condition. Therefore, the region $\mathrm{R} 2$, which does not contain covalent bonds, contributes mostly to the stability and durability of the coating. The multivalent bond strength of non-covalent interactions, in particular hydrogen and coordination bonds, on average outperformed the bond strength possibly assigned to covalent bonds, because many more non-covalent interactions were formed. The dominance of these non-covalent interactions can be even enhanced, once the respective interactions act in a cooperative manner.

A quantification of the energy (area under the force extension curve) for multivalent bonds further supports the finding that interactions of intermediate strength (R2) are most advantageous for stable coatings. For the interaction of the single $\mathrm{PG}_{110}-b-\mathrm{P}\left(\mathrm{Cat}_{5}-\mathrm{Ph}_{5}-\mathrm{A}_{2}\right)$ molecule and the $\mathrm{N}_{3}-\mathrm{PG}_{110}-b-\mathrm{P}\left(\mathrm{Cat}_{5}-\mathrm{Ph}_{5}-\right.$ $\mathrm{A}_{2}$ ) layer on $\mathrm{TiO}_{2}$, we found an average detachment energy of $(608 \pm 569) k_{\mathrm{B}} \mathrm{T}$ at $\mathrm{pH} 6.0,(676 \pm 414) k_{\mathrm{B}} \mathrm{T}$ at $\mathrm{pH} 8.6$ and $(814 \pm$ 868) $k_{\mathrm{B}} \mathrm{T}$ at $\mathrm{pH} 8.6$ crosslinked. These values were higher than

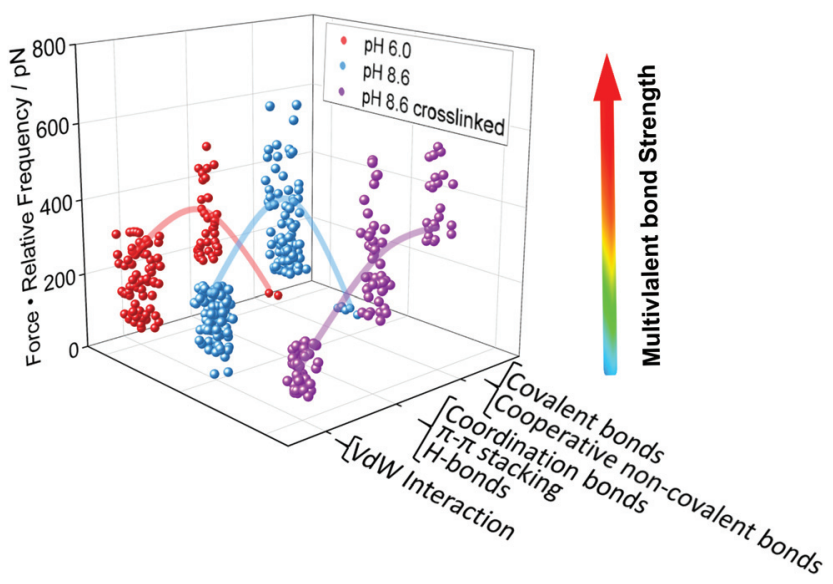

Fig. 4 Multivalent bond strength as basis for the design of polymer coatings. We have quantified the multivalent bond strength, which is the product of the force and the relative frequency of its occurrence, for the $\mathrm{PG}_{110}-b-\mathrm{P}\left(\mathrm{Cat}_{5}-\mathrm{Ph}_{5}-\mathrm{A}_{2}\right)$ molecular force sensor on the $\mathrm{N}_{3}-\mathrm{PG}_{110}-b-\mathrm{P}$ $\left(\mathrm{Cat}_{5}-\mathrm{Ph}_{5}-\mathrm{A}_{2}\right.$ ) layer on $\mathrm{TiO}_{2}$, as given in Fig. 3a. Bonds of intermediate strength showed the highest contribution to the overall strength of the interaction, even in the case of possible covalent bond formation. The lines are a guide to the eye. the energy necessary to break a covalent bond. ${ }^{63}$ In addition, the number of detachment events found in a single forceextension trace reached up to 11 upon crosslinking of the $\mathrm{N}_{3}$ $\mathrm{PG}_{110}-b-\mathrm{P}\left(\mathrm{Cat}_{5}-\mathrm{Ph}_{5}-\mathrm{A}_{2}\right)$ layer (Fig. $\left.\mathrm{S} 9 \dagger\right)$. Furthermore, the average energy per detachment event increases with the number of detachment events per curve $n$. In particular, this increase is strong for $n \geq 4$ for $\mathrm{pH} 8.6$ crosslinked (Fig. S10 $\dagger$ ). This result underlines the increased presence of cooperative non-covalent bonds and also covalent bonds for the crosslinked polymer layer.

Finally, the robustness of a coating comprising a complex multifunctional molecule such as $\mathrm{N}_{3}-\mathrm{PG}_{110}-b-\mathrm{P}\left(\mathrm{Cat}_{5}-\mathrm{Ph}_{5}-\mathrm{A}_{2}\right)$ towards environmental changes is a result of the different interactions present. Once the catechol interaction with $\mathrm{TiO}_{2}$ decreases with increasing $\mathrm{pH}$ value, the possibility of crosslinking of quinone groups to form covalent bonds with amine moieties increases, enhancing the density of the polymer layer, which in turn increases the number of possibly cooperative non-covalent bonds. Thus, in this system, the formation of one type of bond compensates the reduction of another type of bond again demonstrating the power of the interplay between various bond types in a multivalent system.

\section{Conclusion}

Multivalent bonds are important in a wide range of biological systems for, e.g., virus-cell interactions, antigen-antibody interactions or cell-cell signalling. Here, we have performed AFM-based SMFS for the molecular investigation of catecholic, multivalent and heterofunctional polymer layers in order to identify the multivalent bond strengths and their interplay for technical applications. We were able to quantify the force range of a variety of interactions. The formation of vdW interactions, hydrogen bonds, $\pi-\pi$ stacking and coordination bonds could be assigned to detachment events with forces of several 10 to $500 \mathrm{pN}$. A simultaneous occurrence of these non-covalent interactions leads to forces of up to $1500 \mathrm{pN}$. In addition, forces greater than $1500 \mathrm{pN}$ could be attributed to the formation of non-covalent bonds in a cooperative way as well as covalent bonds. The formation of these bonds, which result in highest forces, is enhanced by crosslinking of the polymer layer beforehand. Crosslinking leads to a denser layer, which favours a higher number of multivalent interactions per polymer and area.

Surprisingly, the multivalent bond strength was not highest for covalent bonds but for non-covalent bonds, like hydrogen and coordination bonds, because more bonds of these types could be formed and possibly act in a cooperative manner.

Thus, the combination of non-covalent and covalent interactions, i.e., the multivalency of the presented system, explain the interaction strength obtained by SMFS experiments. Interactions of intermediate strength (non-covalent) take a prominent role among the molecular interactions, even outperforming strong interactions (covalent bonds) under many environmental conditions. In robust and durable coatings, 
non-covalent bonds could therefore serve as sacrificial bonds that can be reformed upon breakage enhancing the longevity of polymer-based coatings. Our findings underline the need of complex molecules for coatings for future applications under varying conditions and provide quantitative values for their design.

\section{Author contributions}

BNB, TH and RH conceived and designed the study. LY synthesized the multifunctional block copolymers. ML and WC performed the experiments and the evaluation of the data with the support of LY, KR, AH, RH, TH and BNB. All authors contributed to the writing of the manuscript and approved its final submitted version. All authors have read and agreed to the published version of the manuscript.

\section{Conflicts of interest}

There are no conflicts to declare.

\section{Acknowledgements}

We thank the Deutsche Forschungsgemeinschaft (DFG, German Research Foundation) under Germany's Excellence Strategy - EXC-2193/1 - 390951807 (ML, TH and BNB) and the collaborative research center SFB 765 (LY and RH) and the grant HU 997/13-1 (WC and TH) for financial support.

\section{References}

1 C. Fasting, C. A. Schalley, M. Weber, O. Seitz, S. Hecht, B. Koksch, J. Dernedde, C. Graf, E. W. Knapp and R. Haag, Angew. Chem., Int. Ed., 2012, 51, 10472-10498.

2 Q. Lin, D. Gourdon, C. Sun, N. Holten-Andersen, T. H. Anderson, J. H. Waite and J. N. Israelachvili, Proc. Natl. Acad. Sci. U. S. A., 2007, 104, 3782-3786.

3 J. Yang, M. A. C. Stuart and M. Kamperman, Chem. Soc. Rev., 2014, 43, 8271-8298.

4 W. Zhang, R. Wang, Z. Sun, X. Zhu, Q. Zhao, T. Zhang, A. Cholewinski, F. K. Yang, B. Zhao and R. Pinnaratip, Chem. Soc. Rev., 2020, 49, 433-464.

5 J. Yang, V. Saggiomo, A. H. Velders, M. A. Cohen Stuart and M. Kamperman, PLoS One, 2016, 11, e0166490.

6 A. H. Hofman, I. A. van Hees, J. Yang and M. Kamperman, Adv. Mater., 2018, 30, 1704640.

7 M. Shin, S.-G. Park, B.-C. Oh, K. Kim, S. Jo, M. S. Lee, S. S. Oh, S.-H. Hong, E.-C. Shin and K.-S. Kim, Nat. Mater., 2017, 16, 147-152.

8 Y. Wang, J. P. Park, S. H. Hong and H. Lee, Adv. Mater., 2016, 28, 9961-9968.

9 G. Zhu, T. Gao, S. Si, Z. Zhang, Q. Liu and G. Zhou, Appl. Surf. Sci., 2020, 507, 145080.
10 Q. Wei, T. Becherer, S. Angioletti-Uberti, J. Dzubiella, C. Wischke, A. T. Neffe, A. Lendlein, M. Ballauff and R. Haag, Angew. Chem., Int. Ed., 2014, 53, 8004-8031.

11 L. Yu, C. Cheng, Q. Ran, C. Schlaich, P.-L. M. Noeske, W. Li, Q. Wei and R. Haag, ACS Appl. Mater. Interfaces, 2017, 9, 6624-6633.

12 L. Yu, Y. Hou, C. Cheng, C. Schlaich, P.-L. M. Noeske, Q. Wei and R. Haag, ACS Appl. Mater. Interfaces, 2017, 9, 44281-44292.

13 H. C. Kolb, M. Finn and K. B. Sharpless, Angew. Chem., Int. Ed., 2001, 40, 2004-2021.

14 M. F. Debets, S. S. Van Berkel, J. Dommerholt, A. J. Dirks, F. P. Rutjes and F. L. Van Delft, Acc. Chem. Res., 2011, 44, 805-815.

15 T. Yamada, C. G. Peng, S. Matsuda, H. Addepalli, K. N. Jayaprakash, M. R. Alam, K. Mills, M. A. Maier, K. Charisse and M. Sekine, J. Org. Chem., 2011, 76, 11981211.

16 G. Pourceau, A. Meyer, J.-J. Vasseur and F. Morvan, J. Org. Chem., 2009, 74, 6837-6842.

17 T. A. Schaub, J. T. Margraf, L. Zakharov, K. Reuter and R. Jasti, Angew. Chem., Int. Ed., 2018, 57, 16348-16353.

18 M. Rief, F. Oesterhelt, B. Heymann and H. E. Gaub, Science, 1997, 275, 1295-1297.

19 B. N. Balzer, M. Gallei, M. V. Hauf, M. Stallhofer, L. Wiegleb, A. Holleitner, M. Rehahn and T. Hugel, Angew. Chem., Int. Ed., 2013, 52, 6541-6544.

20 S. Lv, D. M. Dudek, Y. Cao, M. Balamurali, J. Gosline and H. Li, Nature, 2010, 465, 69-73.

21 W. Cai, D. Xu, L. Qian, J. Wei, C. Xiao, L. Qian, Z.-Y. Lu and S. Cui, J. Am. Chem. Soc., 2019, 141, 9500-9503.

22 M. Grandbois, M. Beyer, M. Rief, H. Clausen-Schaumann and H. E. Gaub, Science, 1999, 283, 1727-1730.

23 P. Schwaderer, E. Funk, F. Achenbach, J. Weis, C. Bräuchle and J. Michaelis, Langmuir, 2008, 24, 1343-1349.

24 S. W. Schmidt, M. K. Beyer and H. Clausen-Schaumann, J. Am. Chem. Soc., 2008, 130, 3664-3668.

25 S. Krysiak, Q. Wei, K. Rischka, A. Hartwig, R. Haag and T. Hugel, Beilstein J. Org. Chem., 2015, 11, 828-836.

26 H. Lee, N. F. Scherer and P. B. Messersmith, Proc. Natl. Acad. Sci. U. S. A., 2006, 103, 12999-13003.

27 G. D. Degen, P. R. Stow, R. B. Lewis, R. C. Andresen Eguiluz, E. Valois, K. Kristiansen, A. Butler and J. N. Israelachvili, J. Am. Chem. Soc., 2019, 141, 1867318681.

28 Y. Mu, P. Mu, X. Wu and X. Wan, Appl. Surf. Sci., 2020, 530, 146973.

29 P. Das and M. Reches, Nanoscale, 2016, 8, 15309-15316.

30 L. Han, L. Gong, J. Chen, J. Zhang, L. Xiang, L. Zhang, Q. Wang, B. Yan and H. Zeng, ACS Appl. Mater. Interfaces, 2018, 10, 2166-2173.

31 P. Steinbauer, A. Rohatschek, O. Andriotis, N. Bouropoulos, R. Liska, P. J. Thurner and S. Baudis, Langmuir, 2020, 36, 13292-13300.

32 Y. Li, H. Liu, T. Wang, M. Qin, Y. Cao and W. Wang, ChemPhysChem, 2017, 18, 1466-1469. 
33 Y. Li, J. Cheng, P. Delparastan, H. Wang, S. J. Sigg, K. G. DeFrates, Y. Cao and P. B. Messersmith, Nat. Commun., 2020, 11, 1-8.

34 T. Utzig, P. Stock and M. Valtiner, Angew. Chem., 2016, 128, 9676-9680.

35 A. Wislez, D. Sluysmans, N. Giamblanco, N. Willet, F. Bano, C. Van De Weerdt, C. Detrembleur and A.-S. Duwez, Biomacromolecules, 2020, 22, 183-189.

36 J. L. Cuellar-Camacho, S. Bhatia, V. Reiter-Scherer, D. Lauster, S. Liese Jr., P. Rabe, A. Herrmann and R. Haag, J. Am. Chem. Soc., 2020, 142, 12181-12192.

37 T. A. Sulchek, R. W. Friddle, K. Langry, E. Y. Lau, H. Albrecht, T. V. Ratto, S. J. DeNardo, M. E. Colvin and A. Noy, Proc. Natl. Acad. Sci. U. S. A., 2005, 102, 1663816643.

38 V. Reiter-Scherer, J. L. Cuellar-Camacho, S. Bhatia, R. Haag, A. Herrmann, D. Lauster and J. P. Rabe, Biophys. J., 2019, 116, 1037-1048.

39 M. Delguste, C. Zeippen, B. Machiels, J. Mast, L. Gillet and D. Alsteens, Sci. Adv., 2018, 4, eaat1273.

40 Q. Hu, H. Yang, Y. Wang and S. Xu, Chem. Commun., 2016, 52, 3705-3708.

41 E. Peterson, C. Joseph, H. Peterson, R. Bouwman, S. Tang, J. Cannon, K. Sinniah and S. K. Choi, Langmuir, 2018, 34, 7135-7146.

42 A. Gomez-Casado, H. H. Dam, M. D. Yilmaz, D. Florea, P. Jonkheijm and J. Huskens, J. Am. Chem. Soc., 2011, 133, 10849-10857.

43 F. Oesterhelt, M. Rief and H. Gaub, New J. Phys., 1999, $1,6$.

44 S. Liese, M. Gensler, S. Krysiak, R. Schwarzl, A. Achazi, B. Paulus, T. Hugel Jr., P. Rabe and R. R. Netz, ACS Nano, 2017, 11, 702-712.

45 J. L. Hutter and J. Bechhoefer, Rev. Sci. Instrum., 1993, 64, 1868-1873.
46 S. Zou, H. Schönherr and G. J. Vancso, J. Am. Chem. Soc., 2005, 127, 11230-11231.

47 S. Kawai, T. Nishiuchi, T. Kodama, P. Spijker, R. Pawlak, T. Meier, J. Tracey, T. Kubo, E. Meyer and A. S. Foster, Sci. Adv., 2017, 3, e1603258.

48 W. Cai, C. Xiao, L. Qian and S. Cui, Nano Res., 2019, 12, 5761.

49 H.-J. Butt, Biophys. J., 1991, 60, 1438-1444.

50 T. Pirzer and T. Hugel, ChemPhysChem, 2009, 10, 27952799.

51 M. Rief, H. Clausen-Schaumann and H. E. Gaub, Nat. Struct. Biol., 1999, 6, 346-349.

52 M. Geisler, S. Xiao, E. M. Puchner, F. Gräter and T. Hugel, J. Am. Chem. Soc., 2010, 132, 17277-17281.

53 F. Kilchherr, C. Wachauf, B. Pelz, M. Rief, M. Zacharias and H. Dietz, Science, 2016, 353, 1116.

54 D. Sluysmans, L. Zhang, X. Li, A. Garci, J. F. Stoddart and A.-S. Duwez, J. Am. Chem. Soc., 2020, 142, 21153-21159.

55 A. Erbas, D. Horinek and R. R. Netz, J. Am. Chem. Soc., 2012, 134, 623-630.

56 J. Blass, M. Albrecht, B. L. Bozna, G. Wenz and R. Bennewitz, Nanoscale, 2015, 7, 7674-7681.

57 X. Zhang and V. T. Moy, Biophys. Chem., 2003, 104, 271278.

58 M. Salomäki, L. Marttila, H. Kivelä, T. Ouvinen and J. Lukkari, J. Phys. Chem. B, 2018, 122, 6314-6327.

59 N. Umek, B. Geršak, N. Vintar, M. Šoštarič and J. Mavri, Front. Mol. Neurosci., 2018, 11, 467.

60 R. Pinnataip and B. P. Lee, ACS Omega, 2021, 6, 5113-5118.

61 T. D. Jacobs and R. W. Carpick, Nat. Nanotechnol., 2013, 8, 108-112.

62 C. Schoeler, R. C. Bernardi, K. H. Malinowska, E. Durner, W. Ott, E. A. Bayer, K. Schulten, M. A. Nash and H. E. Gaub, Nano Lett., 2015, 15, 7370-7376.

63 S. W. Benson, J. Chem. Educ., 1965, 42, 502. 